\title{
A Review of Representation Issues and Modeling Challenges with Influence Diagrams
}

\author{
Concha Bielza \\ Dept. de Inteligencia Artificial, Universidad Politécnica de Madrid, Boadilla del Monte, Madrid 28660, SPAIN \\ mcbielza@fi.upm.es \\ Manuel Gómez \\ Dept. of Computer Science and Artificial Intelligence, University of Granada, Granada 18071, SPAIN \\ mgomez@decsai.ugr.es \\ Prakash P. Shenoy \\ School of Business, University of Kansas, Lawrence, KS 66045, USA \\ pshenoy@ku.edu
}

Since their introduction in the mid 1970s, influence diagrams have become a de facto standard for representing Bayesian decision problems. The need to represent complex problems has led to extensions of the influence diagram methodology designed to increase the ability to represent complex problems. In this paper, we review the representation issues and modeling challenges associated with influence diagrams. In particular, we look at the representation of asymmetric decision problems including conditional distribution trees, sequential decision diagrams, and sequential valuation networks. We also examine the issue of representing the sequence of decision and chance variables, and how it is done in unconstrained influence diagrams, sequential valuation networks, and sequential influence diagrams. We also discuss the use of continuous chance and decision variables, including continuous conditionally deterministic variables. Finally, we discuss some of the modeling challenges faced in representing decision problems in practice and some software that is currently available.

Key words: Decision-making under uncertainty, influence diagrams, probabilistic graphical models, sequential decision diagrams, unconstrained influence diagrams, sequential valuation networks, sequential influence diagrams, partial influence diagrams, limited memory influence diagrams, Gaussian influence diagrams, mixture of Gaussians influence diagrams, mixture of truncated exponentials influence diagrams, mixture of polynomials influence diagrams

\section{Introduction}

Influence diagrams (IDs) are graphical models for representing and solving complex decision-making problems based on uncertain information. Nowadays, they have become a popular and standard modeling tool. As pointed out in a recent special issue of the Decision Analysis journal devoted to IDs, these models "command a unique position in the history of graphical models" [62]. They were first used in 1973 by the Decision Analysis Group at Stanford Research Institute for a project for the Defense Intelligence Agency. IDs were used to model political conflicts in the Persian Gulf to 
see whether more intelligence resources should be allocated, and they tried to measure the value of information produced by the Agency. Miller invented the new graphical convention that we now call an ID. Howard coined the term "influence diagram." The first resulting (classified) paper on IDs is [58]. This information is chronicled by the authors of the facts in [37]. But it is Howard and Matheson's paper, [34], reprinted in [36], that is considered to contain the invention of IDs and to be the pioneering paper. Other interesting details of the evolution and application of IDs since their inception may be found in a retrospective article [35].

IDs are directed acyclic graphs that may be seen as Bayesian networks augmented with decision and value nodes. There are three types of nodes: (1) decision nodes (rectangular) representing decisions to be made; (2) chance nodes (oval or elliptical) representing random variables described by probability distributions; and (3) value nodes (diamond-shaped) representing the (expected) utilities that model the decision-maker's preferences for outcomes. The arcs have different meanings depending on which node they are directed to: the arcs to chance nodes indicate probabilistic dependence, the arcs to value nodes indicate functional dependence, and the arcs to a decision node indicate what information is known at the time the decision has to be made. Accordingly, the arcs are called conditional, functional, and informational, respectively.

At the qualitative (or graphical) level, the ID has a requirement that there must be a directed path comprising all decision nodes. It ensures that the ID defines a temporal sequence (total order) of decisions and it is called the sequencing constraint. As a consequence, IDs have the "no-forgetting" property: the decision maker remembers the past observations and decisions. In contrast, the transitive closure of the precedence binary relation induced by the informational arcs is a partial order on the set of all decision and chance nodes. Therefore, the information constraints represented by these arcs may not specify a complete order. From a semantic viewpoint, it means that the decision-maker may not impose constraints regarding which of two or more chance nodes must precede the others in the decision-making process. For example, after deciding to admit a patient into a hospital, it will not matter which chance variable, "cost of stay" or "risks of being admitted" (infections, contagions, etc.) is the first to occur.

At the quantitative level, an ID specifies the state spaces of all decision and chance nodes: a set of alternatives for each decision variable, and the set of possible values $\Omega_{X}$ of each chance variable $X$. Also, a conditional probability table is attached to each chance node consisting of conditional probability distributions, one for each state of its parents (direct predecessors), and a real-valued function, the utility function, is defined over the states of a value node's parents. Probabilities and utilities represent beliefs and preferences, respectively, of the decision maker. When a problem has a utility function that factorizes additively, each of the additive factors is represented as a value node. 
Figure 1 shows an example of the graphical part of an ID. The problem deals with the oil wildcatter's problem [69]. An oil wildcatter has to decide whether to drill or not at a particular site. He does not know whether the site has oil or not. Before he drills, he has the option of conducting a seismic test which will reveal the seismic structure of the site, which is related to oil volume.

$T$ and $D$ are decision variables, $O$ and $S$ are chance variables, and $v_{1}$ and $v_{2}$ are value nodes. $v_{1}$ is a function of the states of $T, v_{2}$ is a function of the states of $D$ and $O$. The joint utility function is the pointwise sum of $v_{1}$ and $v_{2}$. As in a Bayesian network, the arcs directed to chance nodes such as $S$ mean that the conditional probability attached to $S$ is a function of the states of $O$ and $T$. Finally, the informational arcs directed to $D$ say that at the time the drill decision is made, we know the outcome of $S$ and the decision made at $T$. Since we have a directed path $(T, S, D)$, the $\operatorname{arc}(T, D)$ is implied by the no-forgetting condition and could be omitted. The information constraints specify the (complete) order given by $T, S, D$, and $O$.

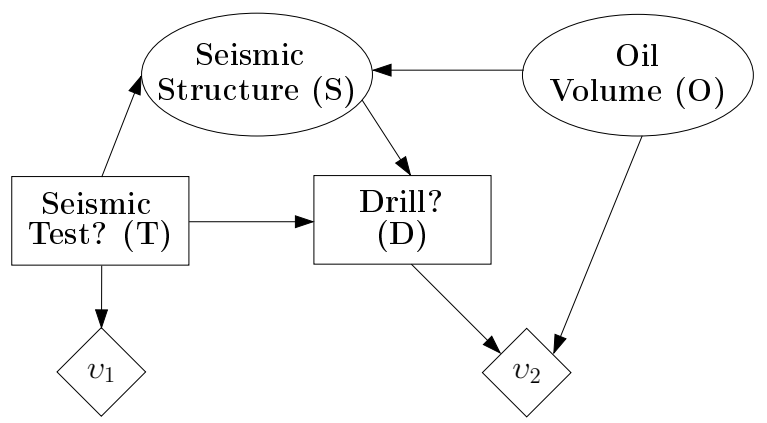

Figure 1: An influence diagram for the oil wildcatter's problem

IDs were initially proposed as a front-end for decision trees, in the sense that their compact representation facilitated the problem structuring, but they were later converted into a decision tree during the solution process. Although there are now several efficient algorithms for solving influence diagrams, many still continue to convert IDs to decision trees during the solution process (see, e.g., [9]). Olmsted [59] describes a method, that involves arc reversals, to solve IDs directly without converting them into decision trees. Shachter [74] published the first ID evaluation algorithm. Evaluating an ID means computing an optimal strategy, one that has the maximum expected utility. Instead of using arc reversals, as in Olmsted's and Shachter's algorithms, other algorithms based on variable elimination strategies or on clique-trees approaches may now be used to solve IDs [18, 78, 38, 77, 90, 52]. Although far from negligible, computational issues related to ID evaluation are beyond the scope of this paper. Some critical difficulties and their solutions are discussed and exemplified in [27, 2], where a large ID, called IctNeo, models neonatal jaundice management for an important public hospital in Madrid.

This paper focuses on modeling issues. IDs have an enormous potential as a model of uncertain 
knowledge. The process of building an ID provides a deep understanding of the problem, and ID outputs are remarkably valuable. Given a specific configuration of variables, an ID yields the best course of action. But ID responses are not limited to provide optimal strategies of the decision-making problem. Inferred posterior distributions may be employed to generate diagnosis outputs (probabilities of each cause). IDs may also automatically generate explanations of their proposals as a way to justify their reasoning $[24,1]$. Moreover, the domain expert may formulate a difficult query, without specifying all the variables required by the ID to determine the optimal decision. It will lead to imprecise responses. If we want the decision maker to receive a convincing response, a refinement is needed, as in [23]. By reasoning in the reverse direction, assuming that the final results of the decisions are known, IDs can be used to generate probabilistic profiles that fit these final results. For example, after a complete remission of a cancer, endoscopically verified by a surgery decision, we can be interested in the probability distributions of other variables, such as patient survival five years following treatment, side effects, etc. Also, the computation of the expected value of information has been shown to play a vital role in assessing the different sources of uncertainty [75].

The aforementioned special issue of Decision Analysis devoted to IDs is a sign of the lively interest in IDs. Boutilier [6] discusses the profound impact that IDs have had on artificial intelligence. As a professional decision analyst, Buede [7] reports on the value of IDs for tackling challenging real decision problems and considers IDs almost as indispensable as a laptop computer. Pearl [62] recognizes the significant relevance of IDs, but he underscores some limitations. First, due to their initial conception with emphasis on subjective assessment of parameters, econometricians and social scientists continue to use the traditional path diagrams, where the parameters are inferred from the data itself. Second, artificial intelligence researchers, with little interaction with decision analytic researchers (in the early 1980s), established conditional independence semantics using the d-separation criterion, and developed their own computational tools. Thus, although IDs are informal precursors to Bayesian networks, the former had a milder influence on automated reasoning research than the latter. Finally, Pauker and Wong [61] show that IDs have disseminated slowly in the medical literature [60, 55], compared to the dominating model of decision trees.

This paper provides a review of ID modeling, discussing the recent contributions. It aims at bringing closer the theoretical and practical developments of IDs to foster their wide use. Also, the analysis identifies challenges and provides insights into the community involved in the design of decision models using IDs.

The paper is organized as follows. Section 2 discusses the representation of asymmetric decision problems. Section 3 discusses the sequencing of decisions and observations that, when partially specified (or unspecified), leads to another kind of asymmetry. Section 4 discusses the use of continuous chance 
and decision variables, including the use of continuous conditionally deterministic variables. Section 5 discusses some challenges of representing decision problems in practice. Finally, section 6 concludes with a summary, discussion, and an outline of issues not discussed in this paper.

\section{Asymmetric Decision Problems}

In this section, we will define asymmetric decision problems and review various techniques that have been proposed in representing such problems based on influence diagrams.

In a decision tree representation, a path from the root node to a leaf node is called a scenario. A decision problem is said to be symmetric if (i) in all its decision tree representations, the number of scenarios is equal to the cardinality of the Cartesian product of the state spaces of all chance and decision variables, and (ii) there exists a decision tree representation of the problem such that the sequence of variables is the same in all scenarios. A decision problem is said to be asymmetric if it is not symmetric.

We will illustrate an asymmetric problem using the reactor problem, which was originally described by [19], and subsequently modified by [4]. An electric utility firm has to decide whether to build $\left(D_{2}\right)$ a reactor of advanced design $(a)$, conventional design $(c)$, or no reactor $(n)$. If the reactor is successful, i.e., there are no accidents, an advanced reactor is more profitable, but it is riskier. Experience indicates that a conventional reactor $(C)$ has probability 0.98 of being successful $(c s)$ and 0.02 of being a failure $(c f)$. On the other hand, an advanced reactor $(A)$ has probability 0.66 of being successful (as), probability 0.244 of a limited accident (al), and probability 0.096 of a major accident (am). If the firm builds a conventional reactor, the profits are estimated to be $\$ 8 \mathrm{~B}$ if it is a success, and $-\$ 4 \mathrm{~B}$ if it is a failure. If the firm builds an advanced reactor, the profits are $\$ 12 \mathrm{~B}$ if it is a success, $-\$ 6 \mathrm{~B}$ if there is a limited accident, and $-\$ 10 \mathrm{~B}$ if there is a major accident. The firm's utility function is linear in dollars. Before making a decision to build, the firm has an option to conduct a test $\left(D_{1}=t\right)$ or not $(n t)$ of the components of the advanced reactor at a cost of $\$ 1 \mathrm{~B}$. The test results $(R)$ can be classified as bad $(b)$, good $(g)$, or excellent $(e)$. Figure 2 shows a causal probability model for $A$ and $R$. Notice that in the probability model, if $A=a s$, then $R$ cannot assume the state $b$. Thus, if the test results are observed to be bad, then an advanced reactor will result in either a limited or major accident (as per the probability model), and consequently, the Nuclear Regulatory Commission will not license an advanced reactor.

Figure 3 shows a decision tree representation of the reactor problem. The computation of the marginal for $R$, and the conditional for $A$ given $R$, have been done using the causal probability model for $A$ and $R$, and this computation is shown in Figure 4. In this figure, the leaves of the tree contain the joint probabilities for $A$ and $R$. The optimal strategy is to do the test; build a conventional reactor 


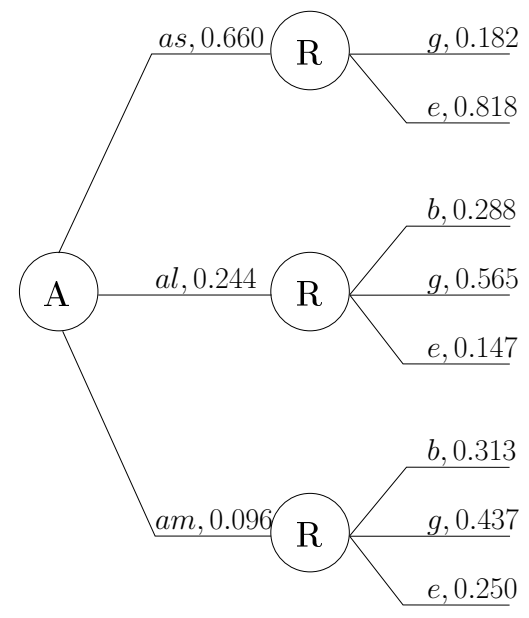

Figure 2: A probability model for $A$ and $R$ in the reactor problem

if the test results are bad or good, and build an advanced reactor if the test results are excellent. The expected profit associated with this strategy is $\$ 8.130 \mathrm{~B}$.

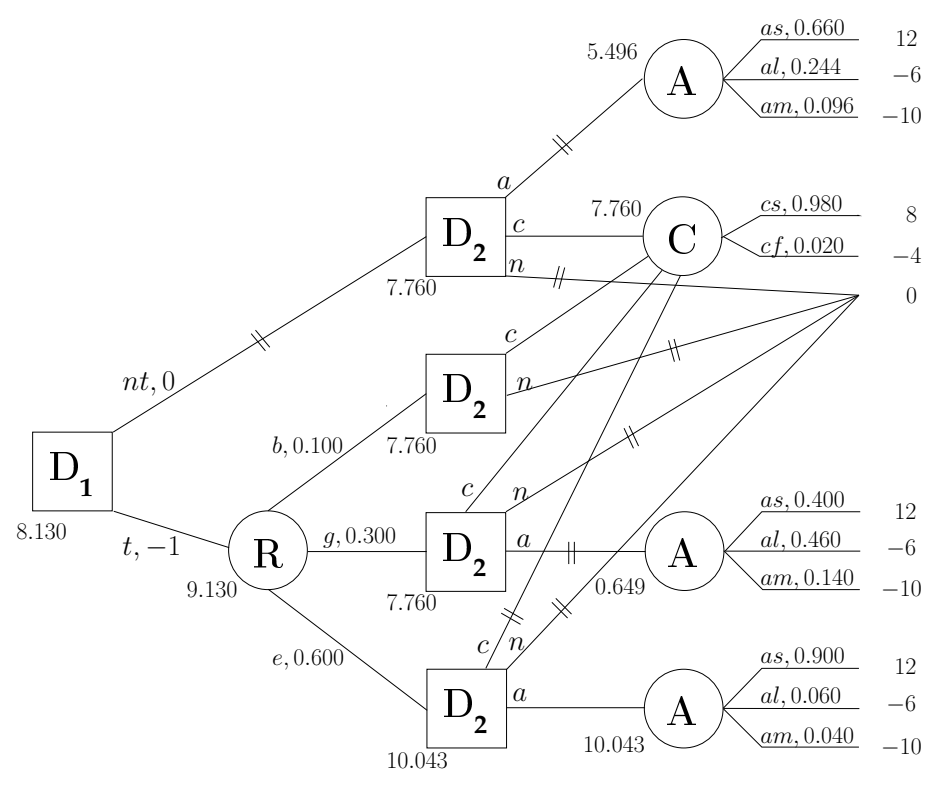

Figure 3: A decision tree representation and solution of the reactor problem

The decision tree representation, shown in Figure 3, easily captures the asymmetric structure of the reactor problem. The cardinality of the Cartesian product of the state spaces of all variables is 108, but there are only 21 possible scenarios. The decision tree is shown using coalescence, i.e., repeating subtrees are shown only once.

There may be three kinds of asymmetry in asymmetric decision problems: chance, decision, and information. The state space of a chance variable may depend on the scenario, and in the extreme, a chance variable may be non-existent. For example, in the reactor problem, $C$ does not occur in scenarios that include $D_{2}=a$. This is called chance asymmetry. Similarly, the state space of a 

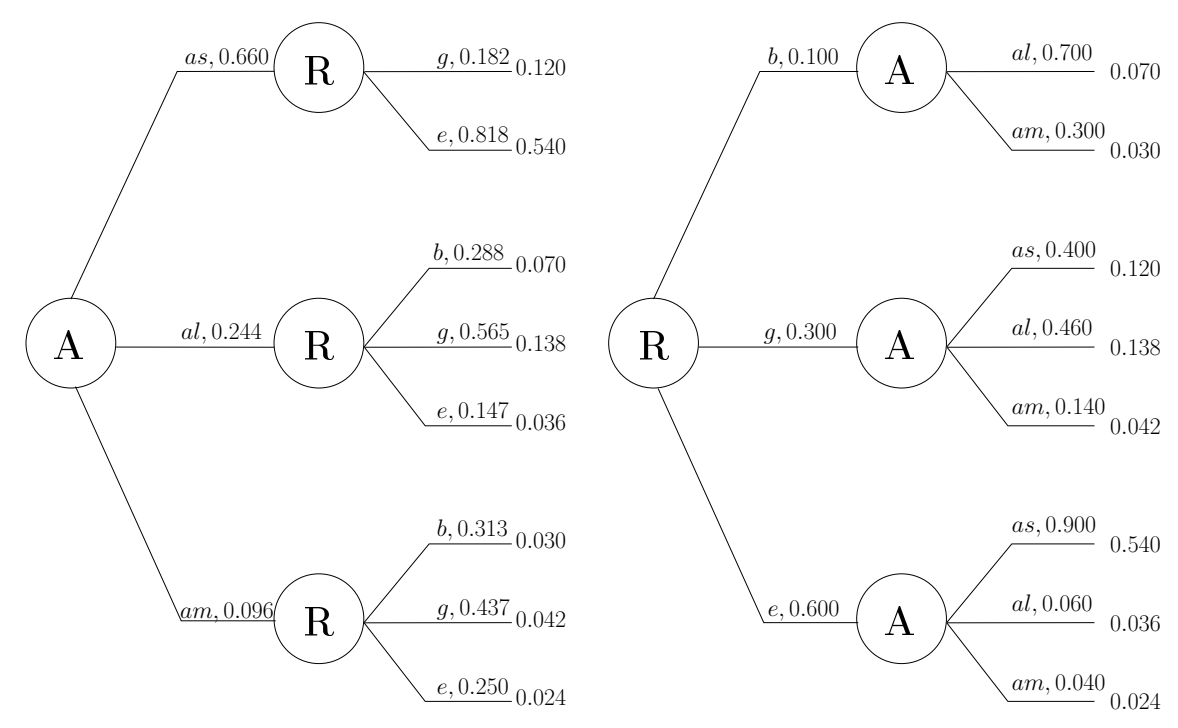

Figure 4: The pre-processing of probabilities in the reactor problem

decision variable may depend on a scenario, and in the extreme, may be non-existent. For example, the alternative $D_{2}=a$ is not available in some scenarios. This is called decision asymmetry. Finally, the sequence of variables may depend on the scenario. For example, $R$ does not precede $D_{2}$ in all scenarios. This is called information asymmetry.

Since decision trees depict all possible scenarios, they can capture asymmetry easily. However, the number of nodes in a decision tree grows combinatorially with the number of variables, and are not tractable for large problems. Also, pre-processing of probabilities may be required (as in the reactor problem).

Influence diagrams were proposed by Howard and Matheson [34] to avoid the problem of preprocessing in decision trees and to represent large problems that would be otherwise intractable using decision trees. However, IDs cannot capture asymmetric features of decision problems as easily as decision trees. One solution is to artificially enlarge the state spaces of chance and decision variables and embed the asymmetric problem in a higher dimensional symmetric problem. For example, an influence diagram representation of the reactor problem is shown in Figure 5.

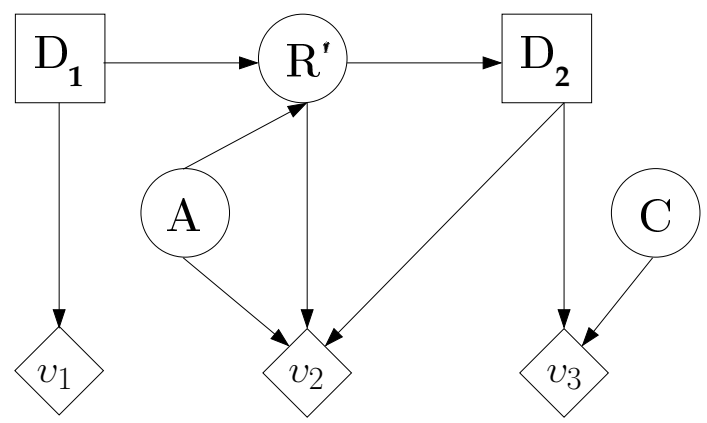

Figure 5: A symmetric ID representation of the reactor problem 
Variable $R$ in the decision tree has been replaced by $R^{\prime}$ which has state space $\{b, g, e, n r\}$, and its conditional distribution now depends also on $D_{1}$ as shown in Table 1 . Thus, if $D_{1}=n t$, then $R^{\prime}=n r$ with probability 1 , and if $D_{1}=t$, then we have the conditional probability distribution as before. Arc $\left(R^{\prime}, D_{2}\right)$ means the true value of $R^{\prime}$ is known when an alternative of $D_{2}$ has to be chosen. $v_{1}, v_{2}$ and $v_{3}$ are additive factors of the joint utility function. The constraint that the alternative $D_{2}=a$ is not available when $R^{\prime}=b$ is modeled by making $R^{\prime}$ a parent of $v_{2}$ and making the value of $v_{2}(b, a, a s)=v_{2}(b, a, a l)=v_{2}(b, a, a m)=-M$, where $M$ is a large positive number. This will ensure that when $R^{\prime}=b, D_{2}=a$ will never be optimal, and the constraint will be satisfied.

\begin{tabular}{ccccccc}
\hline$P\left(R^{\prime} \mid D_{1}, A\right)$ & $(n t, a s)$ & $(n t, a l)$ & $(n t, a m)$ & $(t, a s)$ & $(t, a l)$ & $(t, a m)$ \\
\hline$b$ & 0 & 0 & 0 & 0 & 0.288 & 0.313 \\
$g$ & 0 & 0 & 0 & 0.182 & 0.565 & 0.437 \\
$e$ & 0 & 0 & 0 & 0.818 & 0.147 & 0.250 \\
$n r$ & 1 & 1 & 1 & 0 & 0 & 0 \\
\hline
\end{tabular}

Table 1: The conditional probability distribution for $R^{\prime}$ given $D_{1}$ and $A$

This completes a symmetric influence diagram representation of the reactor problem. Notice that we have increased the size of the problem in the sense that if we convert the influence diagram into a decision tree, it would result in 144 scenarios, whereas the asymmetric decision tree (shown in Figure 3) only has 21 scenarios. Thus, the symmetric influence diagram representation is not very efficient.

To address the inefficiency of the symmetric ID technique, Smith et al. [85] propose representing the asymmetric aspects of a decision problem in the conditional distributions, leaving the overall ID graphical representation unchanged. The conditional distributions are represented using graphical structures called conditional distribution trees consisting of conditioning scenarios and atomic distributions. For example, in the reactor problem, the conditional distribution trees for $D_{2}$ and $R^{\prime}$ are shown in Figure 6.

One advantage of conditioning trees is that the number of scenarios is trimmed back to the minimum necessary. Also, constraints on decision variables can be represented directly without having to add large negative utilities. Thus, we do not need to include an $\operatorname{arc}$ from $R^{\prime}$ to $v_{2}$ in the ID graph. However, some disadvantages remain, such as the artificial state $n r$ for $R^{\prime}$, having $D_{1}$ as a parent of $R^{\prime}$, an arc $\left(D_{1}, D_{2}\right)$ needs to be added to the ID graph, etc. Bielza and Shenoy [4] discuss other strengths, weaknesses and open issues associated with the conditional distribution tree technique.

Next, we will discuss another approach to representing asymmetric decision problems called sequential decision diagrams proposed by Covaliu and Oliver [19]. Unlike the conditioning tree technique, sequential decision diagrams (SDDs) represent the asymmetric structure of the decision problem directly in the SDD graph. The SDD graph for the reactor problem is shown in Figure 7. 

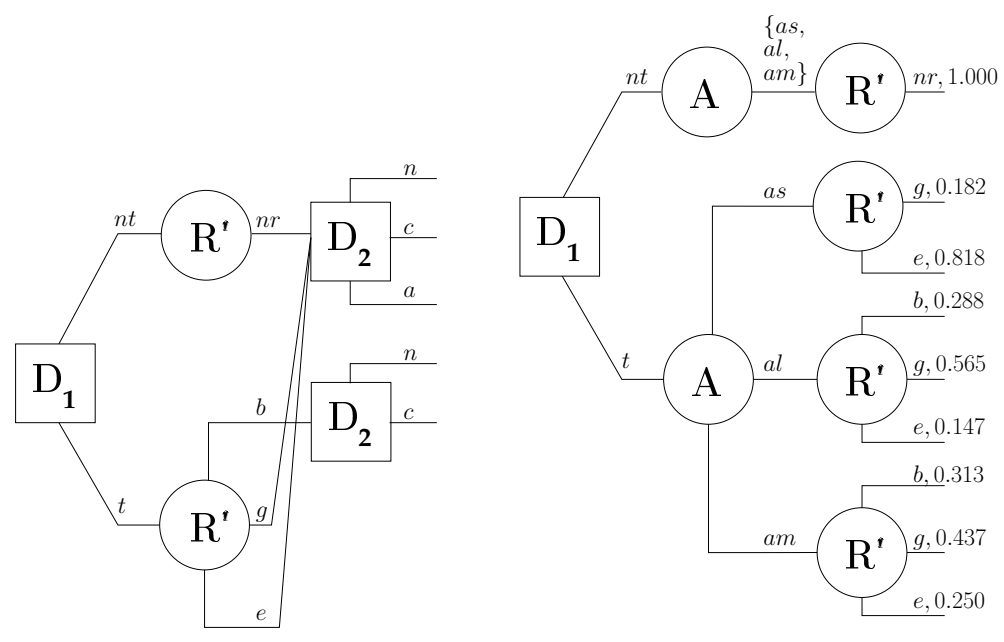

Figure 6: The conditional distribution trees for $D_{2}$ and $R^{\prime}$

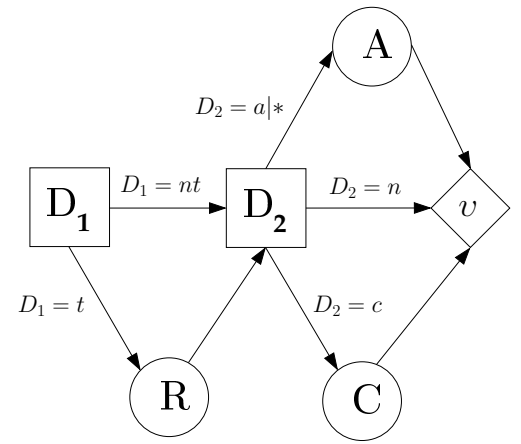

Figure 7: A sequential decision diagram for the reactor problem. $*$ denotes the constraint $\left(D_{1}=\right.$ $n t) \vee\left(\left(D_{1}=t\right) \wedge(R \neq b)\right)$

Like in IDs, each variable appears once in a SDD. The arcs in a SDD denote the sequencing of variables in scenarios like in decision trees. Arcs may be annotated by conditions and constraints. For example, arc $\left(D_{1}, D_{2}\right)$ has a condition $D_{1}=n t$, which means that whenever $D_{1}=n t$, the next variable in the scenario is $D_{2}$. If there are any constraints on decisions, then this can also be listed on the corresponding arc. For example, $D_{2}=a$ is only possible if $D_{1}=n t$, or if $D_{1}=t$ and $R \neq b$. The details of the probability model and utility function are not depicted in the SDD representation. Covaliu and Oliver assume that these details are represented by a corresponding symmetric influence diagram (this would be similar to the symmetric ID in Figure 5, but with only one value node encoding the joint utility function with all five variables as parents).

SDDs can represent the set of all scenarios compactly. Also, we do not need to add dummy states to variables (such as $R=n r$ as ID representations require). On the other hand, there are some drawbacks. First, SDDs cannot represent probability models consistently. For example, the ID representation has state space $\Omega_{R^{\prime}}=\{n r, b, g, e\}$ for $R^{\prime}$, whereas the SDD representation has $\Omega_{R}=\{b, g, e\}$ for $R$. Second, SDD representation assumes that we have an unfactored joint utility function. This implies that solving 
a SDD cannot be done as efficiently (as we could have if we had an additive factorization of the joint utility function).

Demirer and Shenoy [20] propose a representation called sequential valuation networks (SVNs) that is a hybrid of Shenoy's [80] asymmetric valuation network and Covaliu and Oliver's SDDs. A SVN representation of the reactor problem is shown in Figure 8.

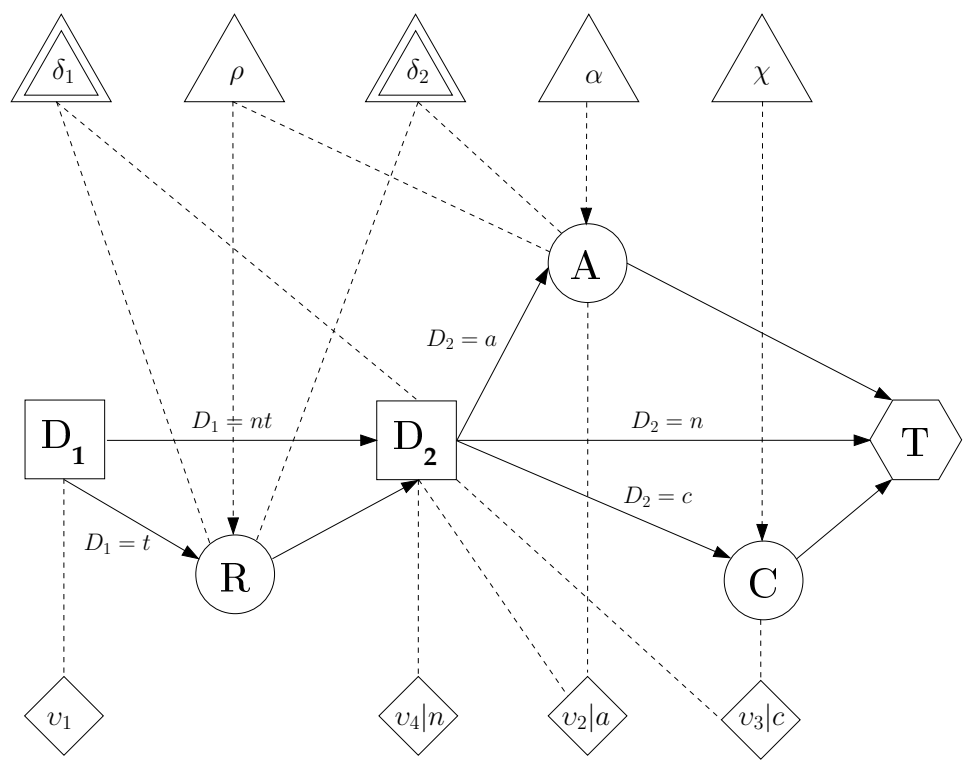

Figure 8: A sequential valuation network for the reactor problem

A SVN consists of an SDD plus indicator, probability and utility valuations. The SVN has an hexagonal terminal node (instead of the diamond node in the SDD) whose only role is to signify the end of a scenario. Solid arcs represent the sequence of variables in scenarios (as in SDDs). Probability valuations are shown as single-bordered triangular nodes. The dashed edges connecting probability valuations to variables denote the domain of the probability valuation. Thus, the domain of $\rho$ is $\{A, R\}$, the domain of $\alpha$ is $\{A\}$, and the domain of $\chi$ is $\{C\}$. If the probability valuation is a conditional for a subset of its domain given the rest of the domain, then the dashed edges to the variables in the subset are directed towards them. Thus, $\rho$ is a conditional for $\{R\}$ given $\{A\}, \alpha$ is a conditional for $\{A\}$ given $\emptyset$, and $\chi$ is a conditional for $\{C\}$ given $\emptyset$. The set of all probability valuations denotes a multiplicative factorization of the joint probability distribution of the chance nodes in the SVN representation. Indicator valuations encode qualitative constraints on the joint state spaces of variables and are represented as double-bordered triangular nodes in the SVN graph. The reactor problem representation has two indicator valuations. $\delta_{1}$ with domain $\left\{R, D_{2}\right\}$ removes the state $(b, a)$ from the joint state space of $\left\{R, D_{2}\right\}$, and $\delta_{2}$ with domain $\{R, A\}$ removes the state $(b, a s)$ from the joint state space of $\{R, A\}$. Utility valuations represent additive factors of the joint utility function and are represented by diamond shaped nodes, and the dashed edges between the utility valuations 
and variables denote the domain of the valuations. In practice, many of the values of the utility factors are zeroes and need not be specified. For example, the costs associated with building an advanced reactor are only associated with $D_{2}=a$. Thus, this factor is defined on the state space $\{a\} \times \Omega_{A}$. This factor is denoted in the SVN representation by $v_{2} \mid a$. Similarly, $v_{3} \mid c$ with domain $\left\{D_{2}, C\right\}$, and $v_{4} \mid n$ with domain $\left\{D_{2}\right\}$ are utility valuations defined on $\{c\} \times \Omega_{C}$ and $\{n\}$, respectively.

Besides the representations already discussed, there are a number of other graphical representations proposed in the literature for asymmetric decision problems. These include combination of symmetric influence diagrams and decision trees [8], decision graphs [68], contingent IDs ([25]), asymmetric valuation networks [80], asymmetric influence diagrams [57], unconstrained influence diagrams [40], coarse valuation networks [51], and sequential influence diagrams [39].

In spite of the plethora of different graphical representations, some aspects of asymmetric decision problems are difficult to represent. One example is a certain event that is repeated a random number of times. Thus, e.g., we can often find medical situations where a specific treatment cannot be performed more than, e.g., twice per full treatment of a patient due to its risks. Also, that risky treatment must be followed and preceded by a certain control treatment, etc. Traditional IDs could not meet these constraints, containing sequences of treatments that are impossible. For another example, a physician has to decide on a subset of possible tests and on a sequence for these tests until a satisfactory diagnosis can be made. If the set of possible tests is large, the combinatorial explosion of the sequencing of all subsets makes the problem intractable for representation and solution. We will discuss this problem in more detail in the next section.

In summary, IDs provide a compact graphical method for representing and solving symmetric decision problems. For asymmetric decision problems, several alternative representations have been proposed, including conditional distribution trees, sequential decision diagrams, and sequential valuation networks. While there have been some studies (e.g., [4]) that compare some of the representations, more studies are needed to compare all of the different proposed representations.

\section{Sequencing of Decisions and Chance Variables}

In this section, we will discuss information constraints that specify the decisions and chance variables that are observed at the time a decision has to be made. It also includes a relaxation of the total order of decisions - sequencing constraint - and those between decisions and chance variables.

In a symmetric decision problem, the information constraints are the same in all scenarios. Thus, in the symmetric ID representation of the reactor problem shown in Figure 5, for decision $D_{1}$ (whether to do a test of the advanced reactor or not), all chance and decision variables are unobserved, and for decision $D_{2}$ (type of reactor to build), the results of the test $R^{\prime}$ and decision $D_{1}$ are observed, but the 
true states of $C$ and $A$ are not. This is represented in the symmetric ID shown in Figure 5 as follows. Since all chance and decision variables are unobserved for $D_{1}$, there are no arrows into $D_{1}$. Since the results of test $R^{\prime}$ are observed for $D_{2}$, we have $\left(R^{\prime}, D_{2}\right)$. Since we have a directed path from $D_{1}$ to $D_{2}$, and we are assuming the no-forgetting condition, the decision made at $D_{1}$ is known when a decision at $D_{2}$ has to be made. This can be modeled explicitly by including an $\operatorname{arc}\left(D_{1}, D_{2}\right)$, or implicitly by not having the arc, but assuming the no-forgetting condition during the evaluation process.

In asymmetric decision problems, the information constraints may not be the same in all scenarios. For example, in the decision tree representation of the reactor problem (shown in Figure 3 ), $R$ is an information predecessor of $D_{2}$ in some scenarios (when $D_{1}=t$ ), but not in some others (when $\left.D_{1}=n t\right)$. While this problem can be made symmetric as described in the previous section, the technique described there - adding artificial states to some chance variables - cannot be applied in all problems. This is especially true if the chance variable being modified is a parent of other chance variables since if we add an artificial state to such a variable, then we have to make up conditional distributions for each of the child variables that are consistent with the original joint distribution, which is a non-trivial task.

Consider a problem of diagnosing diabetes. Diabetes can be detected by doing a blood test and measuring the level of Glucose, which is elevated for diabetic patients. A less expensive test is to take a urine specimen and measure the level of Glucose in urine. A physician is trying to decide whether or not to treat a patient for diabetes. Before she makes this decision, she can order a test (blood or urine). Based on the results of the first test, she can then decide whether or not to order a second test. A symmetric ID model for this problem is shown in Figure 9.

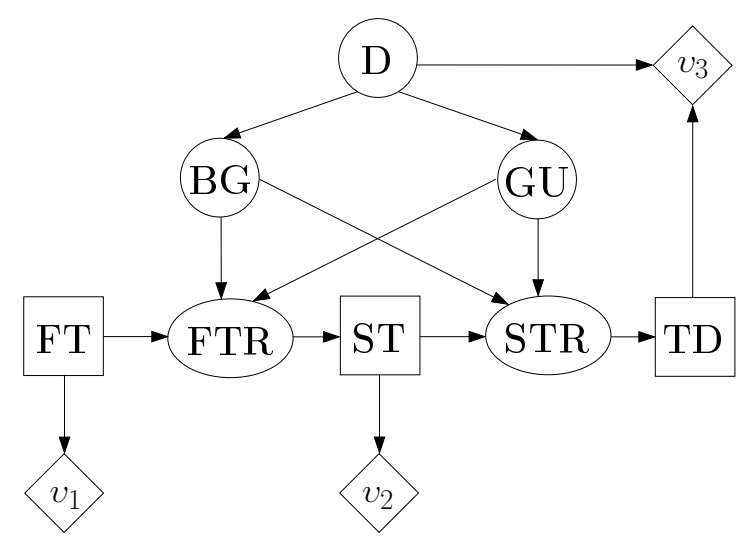

Figure 9: A symmetric ID model for the diabetes diagnosis problem

In this model, $D$ is the chance variable diabetes with states $d$ (has diabetes) and $n d$ (no diabetes), $B G$ is the chance variable blood Glucose with states bge (elevated) and bgn (normal), $G U$ is the chance variable Glucose in urine with states gue (elevated) and gun (normal), FT is the decision variable first 
test with states bt (blood test), ut (urine test), and $n t$ (no test), FTR is the chance variable first test results with states bge, bgn, gue, gun, and $n r$ (no results), ST is the decision variable second test with the same states as FT, STR is the chance variable second test results with the same states as FTR, and $T D$ is the decision variable treatment for diabetes with states $t d$ (treat) and $n t d$ (not treat). $v_{1}, v_{2}$, and $v_{3}$ are additive factors of the joint utility function. FTR and $S T R$ are conditionally deterministic variables with conditional distributions as follows. If $F T=b t$, and $B G=b g e$, then $F T R=b g e$ with probability 1, etc. This formulation allows the possibility of repeating the same test. An advantage of this formulation is that we leave the original Bayesian network consisting of $\operatorname{arcs}(D, B G)$, and $(D, G U)$ unchanged with no artificial states added to the chance variables. A disadvantage of this formulation is that we add two artificial chance variables FTR and $S T R$ with large state spaces. Thus, if we have a decision problem with, say 20 possible tests, formulating a symmetric ID in this fashion would be intractable to solve.

To deal with diagnosis problems with many possible tests and no ordering specified among them, Jensen and Vomlelova [40] propose a representation called unconstrained influence diagrams (UIDs). In UIDs, the requirement that there exists a directed path that includes all decision variables is dropped. The order of tests is deliberately unspecified so that there is a partial order among the decision variables. In the solution phase, we need to determine the tests to be done in some sequence as a function of what is observed from the results of the tests that are already done. A UID model for the diabetes diagnosis problem is shown in Figure 10.

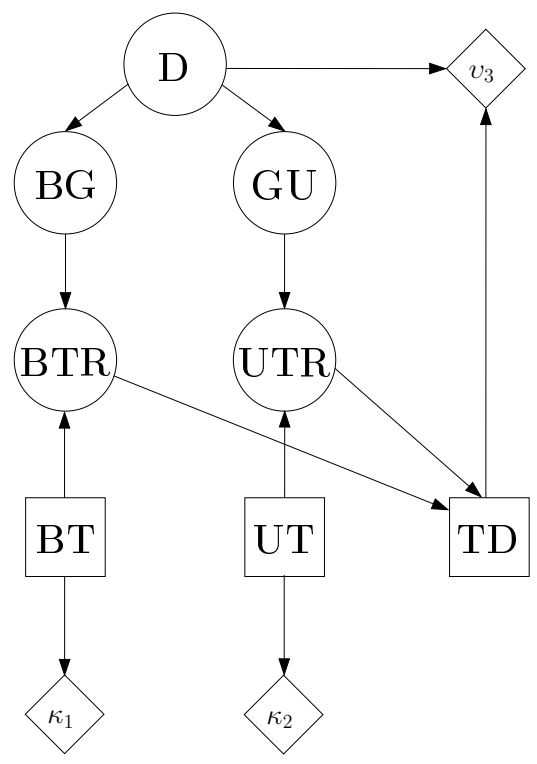

Figure 10: An unconstrained ID model for the diabetes diagnosis problem

In this model, $D, B G, G U, T D$ are as before. $B T$ is a decision variable with states $b t$ and $n t$, and $U T$ is a decision variable with states $u t$ and $n t . B T R$ (for blood test results) is a chance variable with 
states bge, bgn, and $n r$, and $U T R$ (for urine test results) is a chance variable with states gue, gun, and $n r . B T R$ and $U T R$ are conditionally deterministic variables such that if $B T=b t$ and $B G=b g e$, then $B T R=$ bge with probability 1, etc. If $B T=n t$, then $B T R=n r$ with probability 1 (regardless of the state of $B G$ ). Similarly for $U T R$. Notice that there is only a partial order among the decision variables. $\{B T, U T\}$ precede $T D$. The ordering between $B T$ and $U T$ is left unspecified. An optimal solution would include a sequencing of some tests as a function of what is observed from the previous tests performed. If $B T$ is the first test and $U T$ is the second test, then $B T R$ becomes an informational predecessor of $U T$, etc.

An advantage of UIDs is that the state spaces of some of the decision and chance variables are much smaller than in a corresponding symmetric ID. For example, the state spaces of $B T$ and $U T$ have cardinality two, whereas in the symmetric ID representation, FT and $S T$ have cardinality three. Also, the state spaces of $B T R$ and $U T R$ have cardinality three, whereas the corresponding variables $F T R$ and $S T R$ have cardinality five. Furthermore, BTR and UTR each have two parents, whereas the corresponding variables FTR and $S T R$ each have three parents. (We should note here that $B T R$ and $U T R$ have different semantics than FTR and $S T R$. The latter are artificial variables that are constructed to represent the information asymmetry inherent in the decision problem.) These differences in the number of states are even more accentuated with the number of tests. Thus, if we have a diagnosis problem with say 10 possible tests, a UID representation may be tractable for solution even though a symmetric ID is not.

UIDs are designed to simplify the representation of diagnosis/troubleshooting problems, and they are not appropriate for the general class of decision problems. Also, the evaluation procedure for solving UIDs is much more complex than the procedure for solving a symmetric ID since the sequencing of the tests is not specified and has to be determined in the solution phase. When the number of possible tests is large, solving a UID may also be intractable. At this stage, there is no study on the size of diagnosis problems that can be solved using UIDs. As computing power increases, problems that are intractable today may be tractable in the future.

In principle, a decision tree representation should be able to easily represent the asymmetric features of the diabetes diagnosis problem. A drawback would be the size of the decision tree. Since sequential valuation networks are able to represent a decision tree compactly, this representation could be used to represent this problem using no artificial variables. A SVN representation of the diabetes diagnosis problem is shown in Figure 11. In this model, $F T$ and $S T$ are decision variables with states bt, $u t$, and $n t$. The chance variables $D, B G$, and $G U$ have no dummy states. The model allows for repeating the tests.

The diabetes diagnosis problem can also be represented by a sequential influence diagram [39]. A 


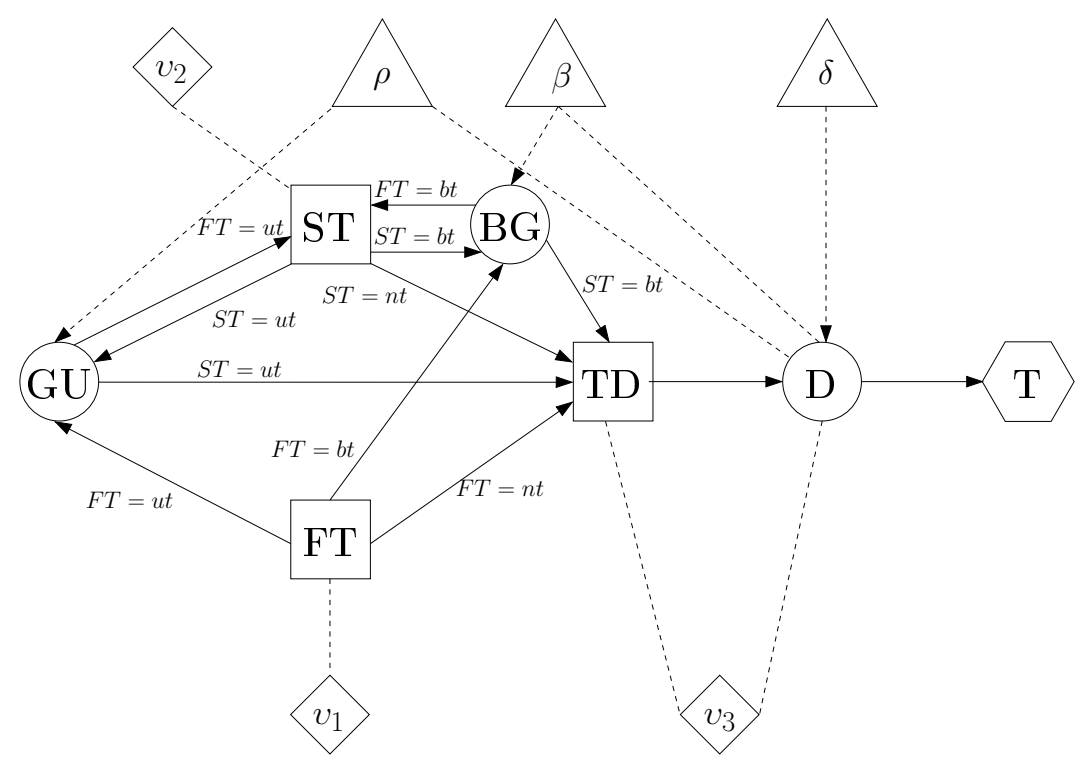

Figure 11: A sequential valuation network model for the diabetes diagnosis problem

sequential influence diagram (SID) can be considered as a combination of sequential decision diagrams, influence diagrams, and unconstrained influence diagrams. There are two types of arcs in a SIDsequential and dependence. To distinguish between the two, we show sequential arcs by solid lines and dependence arcs by dotted lines. If we have a partial order on the information constraints, we use clusters and sequential arcs between clusters to represent partial orders. A SID representation of the diabetes diagnosis problem is shown in Figure 12.

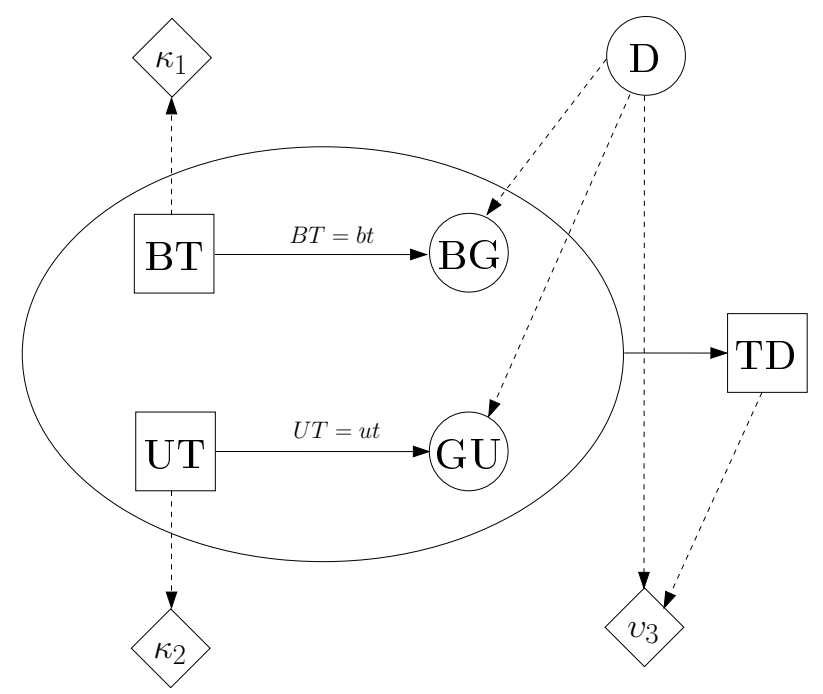

Figure 12: A sequential ID model for the diabetes diagnosis problem

In this model, $\operatorname{arcs}(D, B G)$ and $(D, G U)$ constitute a Bayesian network without any dummy states for $B G$ and $G U$. $B T$ and $U T$ are decision nodes with state spaces $\{b t, n t\}$, and $\{u t, n t\}$, respectively. The sequential arc $(B T, B G)$ with the annotation $B T=b t$ denotes that $B G$ follows $B T$ only in 
scenarios where $B T=b t$. Thus, if $B T=n t$, then $B G$ does not follow $B T$. Similarly for the sequential $\operatorname{arc}(U T, G U)$ with the annotation $U T=u t$. The cluster of nodes containing $B T, U T, B G$ and $G U$ represents the fact that the sequence of tests is unspecified and to be determined. However, the sequential arc from this cluster to $T D$ specifies that in all scenarios, $T D$ follows the two tests. Finally, $D$ follows $T D$ in all scenarios. The remaining part of the diagram has the same semantics as an influence diagram.

If we compare this model with the UID model in Figure 10, we notice that we do not need dummy variables BTR and UTR. If we compare the SID model with the SVN model shown in Figure 11, we notice that the sequence of tests is represented explicitly in the SVN representation, while it is represented in the SID representation using the UID convention. Thus, if we have 10 different tests, a SVN representation can get complex to account for all possible sequences of tests, whereas the SID representation remains simple. The SID representation is more efficient than either the UID representation or the SVN representation. Unlike UIDs, SIDs are designed for representation of the general class of decision problems, not just diagnosis/troubleshooting problems.

We conclude this section with a discussion of partial influence diagrams (PIDs) and limited memory influence diagrams (LIMIDs). The algorithms for solving IDs assume that there is a total ordering of the decision variables. This condition is sufficient for computing an optimal strategy, but it is not necessary. Nielsen and Jensen [56] define partial IDs (PIDs) as IDs where there is no total order specified for decision variables. Since the solution to a decision problem may depend on the ordering of the decision variables, Nielsen and Jensen specify conditions that ensure that a PID has a well-defined optimal strategy even though there is no total order on the decision variables. The conditions are based on d-separation and can be read from the graphical structure of a PID.

Lauritzen and Nielsen [47] introduce the notion of limited memory IDs (LIMIDs) as a model for multistage decision problems in which two conditions are relaxed: total order for decision variables and no-forgetting. The sequence in which decisions are to be made is not specified other than through it being compatible with the partial order induced by the ID graph, i.e., if $D_{2}$ is a descendant of $D_{1}$, then decision $D_{1}$ must be made before $D_{2}$. The parents of a decision node $D$ represent exactly the variables whose values are known and taken into consideration when a decision at $D$ has to be made. Thus, LIMIDs allow for multiple agents of a decision maker (who may not be able to communicate with each other) or for forgetful decision makers.

The example of a LIMID presented in [47] describes a pig breeder problem involving decisions about injecting a certain drug as a treatment for a given disease. The disease can be detected with a test $\left(t_{i}\right)$. The decisions about injections must be done during the first three months of pigs life, one per month $\left(D_{i}, i=1,2,3\right)$. The pigs will be sold in the fourth month. The market price will depend on the 
health status of the pigs $\left(h_{i}\right)$. This problem can be considered with two different points of view. The first one assumes that the breeder keeps individual records for every pig, and this information is used before deciding about the injections. Thus, the no forgetting condition is assumed, see Figure 13(a). The second assumes that there are no individual records for the pigs, and that the decisions are made knowing the test result only for the given month (the LIMID version, see Figure 13(b)). Nodes $v_{i}, i=1,2,3$ are related to the costs of performing the test and $v_{4}$ denotes the benefit obtained with the pig sale.

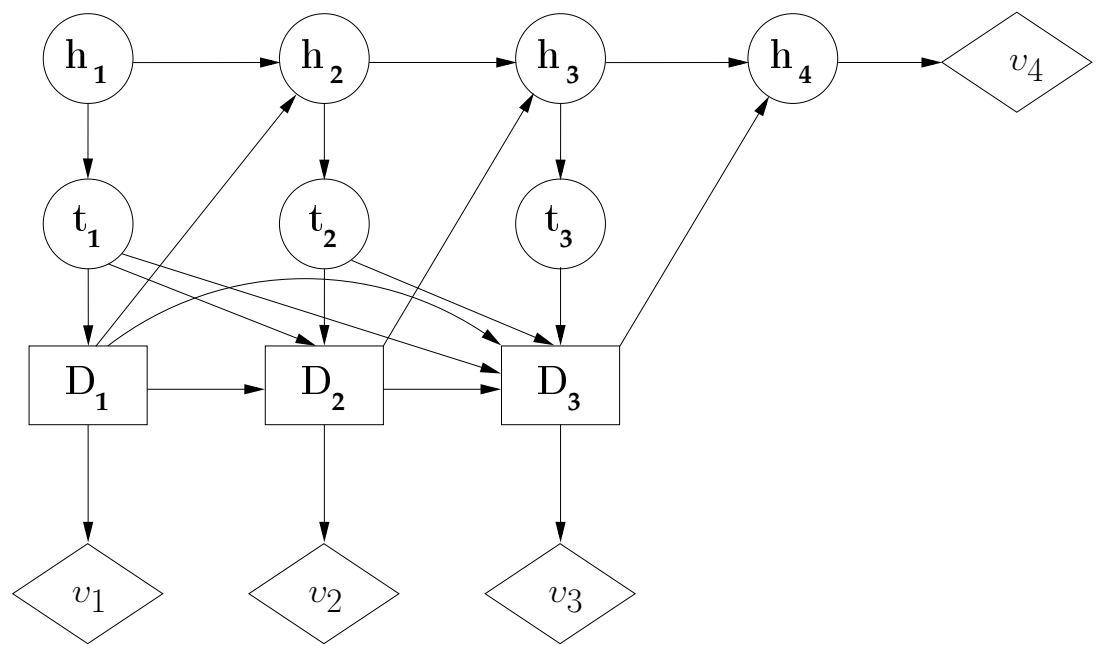

(a) ID version

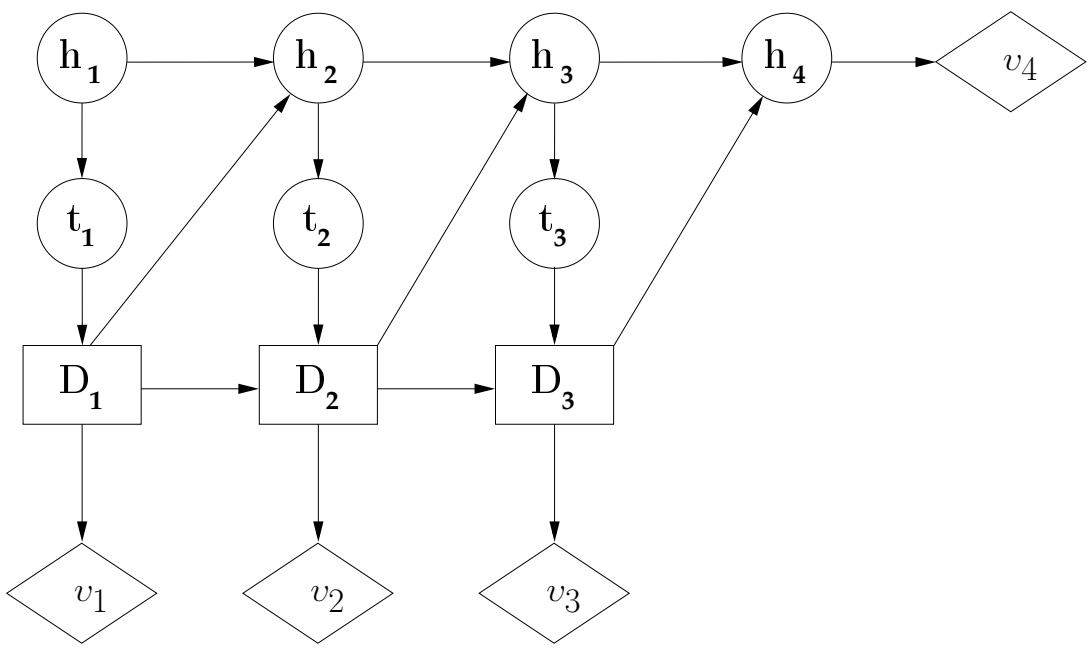

(b) LIMID version

Figure 13: The ID and limited memory ID models for the pig problem

The main motivation of LIMIDs is that by limiting the number of information arcs, its solution is computationally tractable whereas the same decision problem could be intractable if we assumed the no-forgetting condition. The disadvantage is that an optimal solution of a LIMID may not be optimal for the same decision problem if we were to assume the no-forgetting condition. Thus, we trade-off tractability of solution with optimality. Lauritzen and Nilsson describe an algorithm called 
single policy updating for finding a local optimal solution of a LIMID. Thus, if we consider the ID in Figure 10 as a LIMID, and solve it using the single policy updating algorithm, assuming that no test pays by itself, a solution may be that no test shall be done, even though an optimal UID solution for the problem is to first do a urine test, and if positive, then do a blood test.

In summary, we have examined different representations of problems in which we have information asymmetry. We have examined symmetric influence diagrams, unconstrained influence diagrams, sequential valuation networks, sequential influence diagrams, partial influence diagrams, and limited memory influence diagrams. For the class of problems in which we have several diagnostic tests that can be done in any sequence, the sequential influence diagram representation is very efficient in representing such problems. The exact solution of these problems, however, is hard and may not be tractable when we have a large number of tests. In some decision problems, there may not be a total order specified among the decision nodes, but it admits a well-defined optimal solution through the use of partial influence diagrams. Also, for a class of multi-stage problems, influence diagrams may be intractable. By dropping the no-forgetting condition, the solution of a decision problem may be tractable using the single policy updating algorithm of LIMIDs.

\section{Continuous Chance and Decision Variables}

In this section, we review the literature on the use and issues related to using continuous chance and decision variables in representing and solving decision problems.

In practice, it is safe to assume that one encounters chance and decision variables that are continuous. A chance or decision variable is said to be discrete if the state space is countable, and continuous otherwise. Typically, the states of discrete variables are symbols, whereas the states of continuous variables are real numbers. The conditional distribution of continuous chance variables are typically conditional probability density functions (PDFs). One major problem associated with solving IDs with continuous chance variables is integration of products of conditional PDFs and utility functions when computing expected utilities. There are many commonly used PDFs, such as the Gaussian PDF, that cannot be integrated in closed form. Another major problem is finding the maximum of a utility function on the state space of a continuous decision variable. Depending on the nature of the multidimensional utility function, we can have a difficult non-linear optimization problem. To avoid these difficulties, one standard approach is to discretize the continuous variables using bins. Using many bins results in a computational burden for solving the discrete ID, and using too few bins leads to an unacceptable approximation of the problem.

One of the earliest work on using continuous variables in IDs is by Kenley and Shachter [42, 76]. In their representation, which they call Gaussian IDs (GIDs), all chance variables are continuous having 
the so-called conditional linear Gaussian (CLG) distribution. This is a Gaussian PDF, whose mean is a linear function of its parents, and the variance is a constant. An implication of this condition is that the joint distribution of all chance variables is multivariate normal. One can find marginals of the multivariate normal distribution without doing any integration. So the problem of integration does not exist for such IDs. GIDs also assume that all decision nodes are continuous and that the utility function is a quadratic function of its parent chance and decision variables. An implication of this condition is that there is a unique maximum for the decision variables, which can be found in closed form. Thus, the optimization problem of finding an optimal solution does not exist either for such IDs.

An example of a GID is shown in Figure 14 [76]. A consultant has purchased an expensive computer for use in her practice. She will bill her clients for computer usage at an hourly rate. Also, she expects to have the computer unused most of the time and she would like to sell some of these idle hours to timesharing users. She must decide on a price for her consulting clients, and a price for her time-sharing users, so as to maximize her total profit.

She believes that the number of consulting hours sold will depend on consulting price, and that the cost of the computer facilities for consulting, consulting cost, will depend on consulting hours. Her accountant will work up a consulting estimate of the number of consulting hours she will bill. This will be known before deciding on the price for time-sharing users. The number of time-share hours will depend on time share price and the hours the computer is not busy with consulting work (idle hours). The cost of running the time-sharing service, time sharing cost, will depend on the hours purchased, time share hours, and idle hours. Her profit will be the difference between total revenues and costs. Specific parameters for the Gaussian distributions attached to all the continuous variables must be assessed. These are shown in Figure 14, with the corresponding conditional mean and conditional variance next to each node. All continuous variables (chance or decision) are depicted with a double border.

Poland [64] extends GIDs to mixture of Gaussians IDs (MoGIDs). In MoGIDs, we have discrete and continuous chance variables. As in GIDs, continuous variables have a CLG distribution whose mean is a linear function of its continuous parents, and a constant variance. The linear function and constant variance can be different for each state of its discrete parents. However, discrete nodes cannot have continuous parents. An implication of these conditions is that given a state of each discrete variable, the joint distribution of the continuous variables is multivariate normal. Again the problem of integration does not exist. MoGIDs have the same restriction as GIDs regarding utility functions associated with continuous decision variables (quadratic).

An example of a MoGID is shown in Figure 15 [64]. This is a different formulation of the oil wildcatter's problem than the one given earlier in Figure 1. The previous formulation discretizes oil 


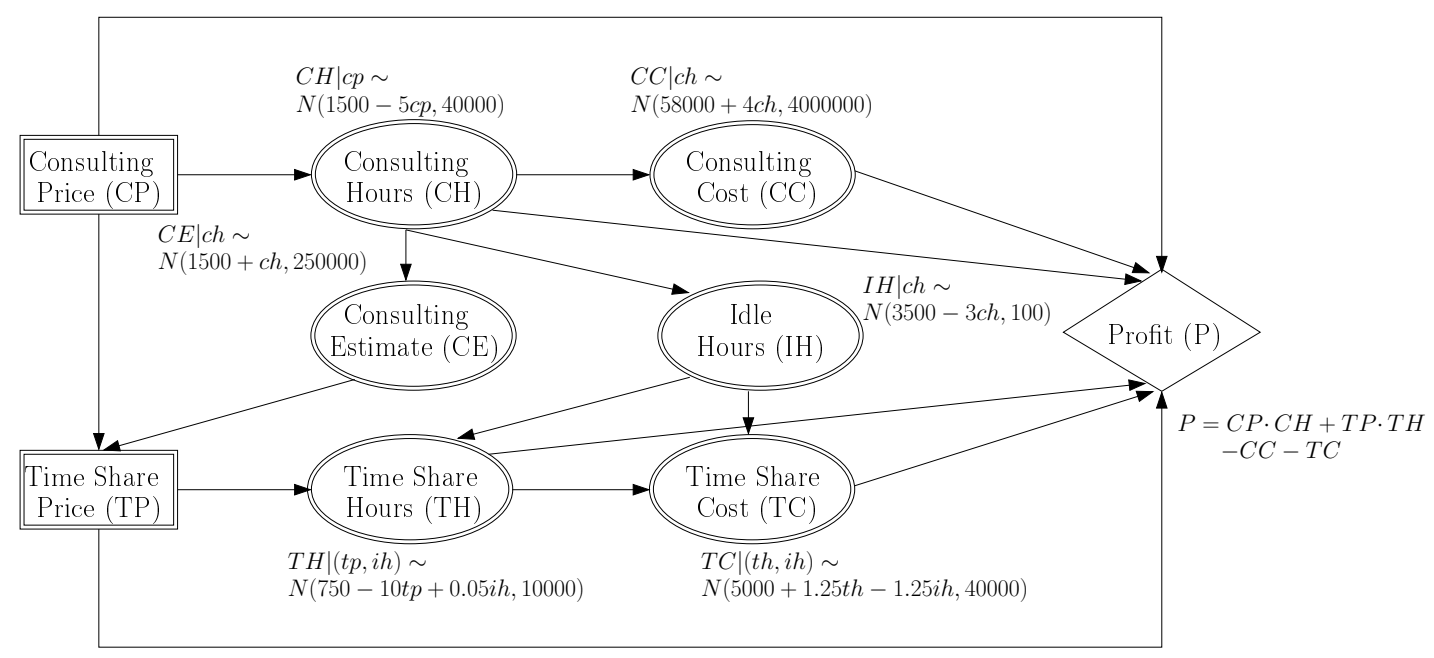

Figure 14: A Gaussian ID for the Consultant's Problem

volume and drilling cost (included in $v_{2}$ ). Here, they are represented as continuous variables (with double borders). Oil volume has a mixed distribution: it has a PDF for positive volumes but there is a probability mass at zero. Poland includes these two variables conditioned by "case" (discrete) variables to make their PDF mixtures. For example, oil volume case has three states: "zero", "medium" and "high", with associated probabilities conditioned on seismic structure. For each of these, oil volume has a distinct distribution: a probability mass at zero for the "zero" case, and a PDF for the other cases.

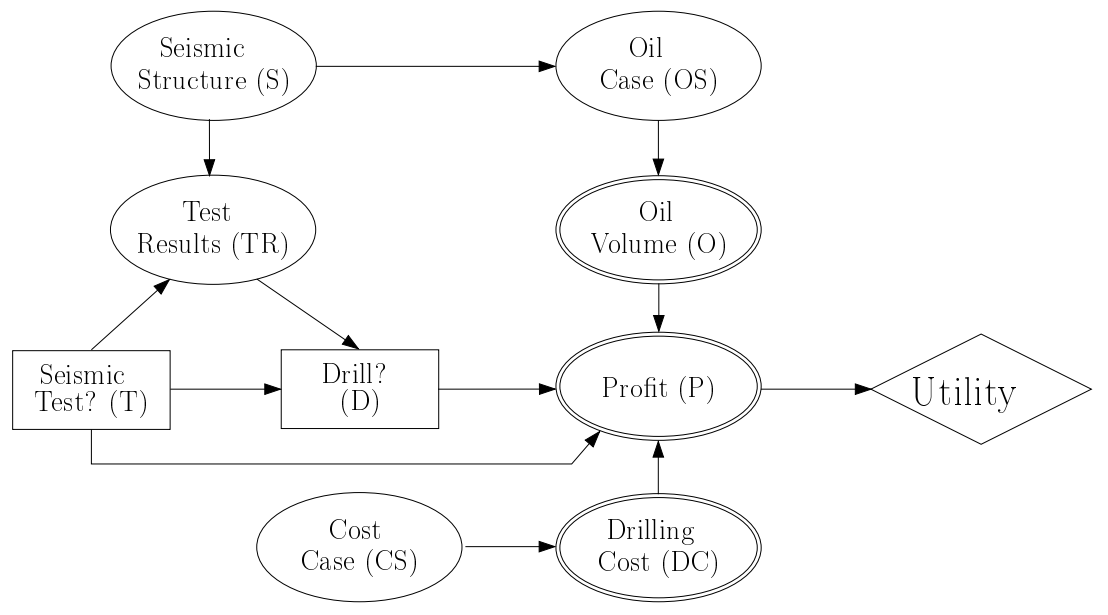

Figure 15: A MoGID for the oil wildcatter's problem

If we have a continuous variable whose conditional PDF is not CLG, then we can approximate it with a mixture of Gaussians. The parameters of the mixture distribution (number of distributions, weights, and means and variances of the Gaussian distributions) can be found either by using a minimum entropy technique [65] or by solving a non-linear optimization problem that minimizes some distance measure between the target distribution and mixture distribution [81].

If we have a discrete chance variable with a continuous parent, then we do not have a mixture 
of multivariate normal distribution for the continuous chance variables. Some authors suggest approximating the product of the conditionals for the discrete variable and its parents by a CLG using a variational approach [54] or a numerical integration called Gaussian quadrature [49]. Shenoy [81] suggests using arc reversals to remedy this situation. However, arc reversals may destroy the CLG distribution of the parent, in which case, it has to be approximated by a mixture of Gaussians.

If besides continuous chance variables, we have a decision problem with (few) continuous decision variables, Bielza et al. [3] suggest the use of a Markov Chain Monte Carlo (simulation) method for finding an approximately optimal strategy as a remedy for the optimization and integration problem. They define an artificial distribution on the product space of chance and decision variables, such that sampling from this artificial augmented probability model is equivalent to solving the original decision problem. The approach can accommodate arbitrary probability models and utility functions.

Charnes and Shenoy [10] investigate the problem of solving large IDs with continuous chance variables and discrete decision variables using a Monte Carlo method where only a small set of chance variables are sampled for each decision node. Using this technique, they solve a problem of valuation of a Bermudan put option with 30 discrete decision variables and continuous chance variables with non-CLG distributions.

Another strategy for easing the problem of integration is suggested by Moral et al. [53]. They suggest approximating conditional PDFs by mixtures of exponential functions whose exponent is a linear function of the state of the variable and its continuous parents. Each mixture component (or piece) is restricted to a hypercube. One advantage of this approximation is that such mixtures of truncated exponentials (MTEs) are easy to integrate in closed form. Also, the family of MTE functions are closed under multiplication and integration, operations used in solving influence diagrams. Cobb et al. [17] describe MTE approximations of commonly used PDFs using an optimization technique similar to the one described earlier for finding parameters of a mixture of Gaussian approximation. Cobb and Shenoy [16] define MTE IDs, where we have continuous chance variables with MTE conditional PDFs, discrete decision variables, and utility functions that are also MTE functions. Such MTE IDs can be solved using the solution technique of discrete IDs with integration used to marginalize continuous chance variables. Cobb [13] introduces continuous decision MTE influence diagrams (CDMTEIDs), where besides using MTE potentials to approximate PDFs and utility functions, continuous decision variables are allowed. A piecewise-linear decision rule for these continuous decision variables is developed.

In the same spirit as MTEs, Shenoy and West [82, 84] suggest the use of mixture of polynomials (MOPs) to approximate conditional PDFs. Like MTE functions, MOP functions are easy to integrate, and the family of MOP functions is closed under multiplication and integration. Unlike MTEs, finding MOP approximations is easier for differentiable functions as one can use the Taylor series expansion 
to find a MOP approximation. Finding an MTE approximation for a multi-dimensional distribution (such as the conditional for a variable given another continuous variables as parents) can be difficult, whereas finding a MOP approximation is easier at it can be found using the multi-dimensional version of the Taylor series.

In an ID, a continuous chance variable is said to be deterministic if the variances of its conditional distributions (for each state of its parents) are all zeroes. An example of a deterministic variable is a continuous variable whose state is a deterministic function of its continuous parents, and the function may depend on the state of its discrete parents. An example of a deterministic variable is Profit $=$ Revenue - Cost, where Revenue and Cost are continuous chance variables, and Profit is a deterministic variable with Revenue and Cost as parents. Deterministic variables pose a special problem since the joint density for all the chance variables does not exist. For GIDs and MoGIDs, deterministic variables are not a problem as long as the functions defining the deterministic variables are linear. The theory of multivariate normal distributions allows linear deterministic functions [71, 46]. The class of MTE functions is also closed under transformations required by linear deterministic functions [14], but not for non-linear deterministic functions. For non-linear deterministic functions, Cobb and Shenoy [15] suggest approximating a non-linear deterministic function by a piecewise linear function, and then using the technique proposed in [14]. Cinicioglu and Shenoy [11] describe an arc reversal theory for hybrid Bayesian networks with deterministic variables with a differentiable deterministic function. They use Dirac delta functions [22] to represent deterministic functions. The deterministic function does not have to be linear or even invertible. As long it is differentiable and its real zeroes can be found, arc reversal can be described in closed form. They conjecture that Olmsted's arc-reversal theory [59] for solving discrete IDs can be used for hybrid IDs using their arc-reversal theory. This claim needs further investigation. The family of MOP functions are closed for a larger class of deterministic functions than MTEs. For example, MOP functions can be used for quotients [82]. Li and Shenoy [50] describe a further extension of the extended Shenoy-Shafer architecture [83] for solving influence diagrams containing discrete, continuous, and deterministic variables. In problems where no divisions need to be done, MOP approximations can be used for PDFs, utility functions, and deterministic functions to alleviate the problems of integration and optimization. Finally, deterministic variables pose no problems for Monte Carlo methods, such as [10], that use independent and identically distributed samples. However, Markov chain Monte Carlo methods may not converge in the presence of deterministic variables.

We conclude this section by remarking that much research remains to be done to make the solution of IDs with continuous chance and decision variables viable in practice. It would be very useful to have a formal comparison of the various techniques on a test bed of problems with different sizes and 
complexity. This includes a comparison of MoGIDs, MTE IDs, and MOP IDs. Also, for an individual technique, such as, e.g., MTE IDs, there are many ways to approximate a PDF by an MTE function. There is a tradeoff between number of exponential terms and number of pieces (mixture components). Fewer pieces invariably imply more exponential terms. It is not known which approximation is the best from a computational viewpoint. A similar issue exists for MoGIDs and MOP IDs. Finally, it would be useful to have bounds on the optimality of the solution based on bounds on the approximations of the PDFs.

\section{Modeling with IDs: Strengths and Limitations}

The previous sections have presented ID modeling capabilities and challenges mostly developed and identified by researchers and published in scientific forums. But there is not much feedback from analysts and experts about their experiences with IDs for building decision-making models. Specifically, it would be interesting to know the main problems perceived by those who have tried to model complex decision-making problems using IDs. Unfortunately, it is well known that the modeling step is not yet automated, and is considered as an art, and in which most of the literature has taken little interest [12]. Perhaps this is the reason why there are very few papers offering this point of view. Two examples of these are $[2,27]$.

These papers describe the problems faced while modeling a neonatal jaundice management problem in the medical domain. This problem is present during the first few days of a baby's life, the first 72 hours after birth being the most critical. The first decision is whether to admit (or not) a baby to a hospital and confining it, eventually, to an intensive care unit. In case the baby is admitted, it is necessary to control the bilirubin levels, carrying out different tests, and applying some of the prescribed treatments: phototherapy, exchange transfusion, or observation. The treatment will be selected depending on some crucial factors such as age, weight, and bilirubin and hemoglobin levels. Treatments are given along several consecutive stages, observing after each one their effects on the baby and repeating the process as many times as necessary until the problem is solved (the infant is then discharged or ( $\mathrm{s}$ )he receives a treatment related to another disease).

The two main problems identified when constructing an ID for this disease are related to time modeling and the existence of constraints between the treatments (both are closely related). Since experts consider that there must be at least 6 hours between two successive treatments (in order to be able to observe their effects), the initial approach considers an ID containing a sequence of 12 decision nodes (one per stage). As treatment decisions depend at each stage on the same set of factors, the

entire model can be considered as a repetition of 12 identical slices, the $i^{\text {th }}$-slice inheriting information from the $(i-1)^{t h}$-slice. All decision nodes would be identical containing the same set of alternatives. 
Moreover, during the modeling stage, several constraints were identified: not to perform more than two exchanges per full treatment, start with observation or phototherapy, precede and follow exchanges by phototherapies, etc. These constraints lead to a highly asymmetric ID and it cannot be modeled with this structure of 12 identical decisions. Another problem related to time management stems from the variable length of the full process. Some patients will need only one treatment stage, for example. For that reason the subsequent decision domains must be filled in with dummy therapeutic actions (do nothing) in the spirit of symmetric IDs. Although direct, this first approximation entails a highly asymmetric and intractable ID due to the considerable set of nodes and arcs.

A deeper analysis revealed that the experts normally consider combinations of therapies: phototherapy of 12 or 18 hours long, for example. Using this fact, and in order to reduce the number of decisions, three types of frames are considered. The first one corresponds to the first decision, containing the alternatives allowed when starting the treatment. The second type will be used for two decision nodes articulating the central part of the treatment. Its alternatives consider grouped treatments satisfying the constraints (e.g., a 12-hour phototherapy followed by an exchange transfusion and then a 12-hour phototherapy again). The fourth and fifth decisions are related to the third type of frame. The fourth one concerns light treatments and the last only final actions. The consideration of combined actions makes more complex the definition of the decision domains, but reduces the model size to five decision nodes and limits the presence of incoherent treatments as well. There is another set of constraints not included in the new domains (because they affect the entire process) and will have to be considered during the model evaluation process.

In spite of these difficulties, the modeling of this ID was described as a rich exercise for experts, who felt very comfortable reasoning about the different alternatives under consideration. This reasoning process led to a better understanding of the jaundice problem, and as a direct consequence, to a drastic reduction in the number of aggressive treatments. These positive outcomes were mostly a result of the modeling process, rather than of the model evaluation process.

Another way to check whether the ID methodology is really useful in practice is to examine the literature on applications of decision problems using IDs. A compilation can be found in [26]. But this examination does not provide information about which systems are real applications and which are just prototypes. Real applications will refer to final systems in use and prototypes may be related to research projects without a commercial use.

More indicators of ID dissemination and acceptance can be obtained by looking at commercial software for modeling and evaluating IDs. These tools would not exist if IDs were not considered useful. As a sample, we list several relevant tools including the URL of the companies. Several of these URLs also contain links to case studies related to IDs, as well as a list of clients or partners who use 
IDs for modeling their decision-making problems.

- Analytica (http://www.lumina.com/) is a visual tool for creating, analyzing, and communicating decision models as IDs.

- Decision Manager (http://www.deciware.com) offers tools for effective business management including decision trees and IDs.

- DPL 7 (http://www.syncopation.com), a professional tool for decision analysis. It offers a single and coherent application with a straightforward graphical user interface.

- HUGIN (http://www.hugin.com) has focused on delivering advanced solutions for decision making under uncertainty. It offers a complete and intuitive tool for building and analyzing BNs and IDs.

- Netica (http://www.norsys.com/) is a powerful and easy-to-use program for working with BNs and IDs.

- PrecisionTree (http://www.palisade.com) does decision and sensitivity analysis in Microsoft Excel using decision trees and IDs.

- TreeAge Pro (http://www.treeage.com) enables the creation of decision trees, IDs and Markov models which can be analyzed with a sophisticated set of tools and offering a variety of graphs, charts and reports for communicating the results.

There are also some free tools with remarkable features. One of them is GeNie (http://genie.sis.pitt.edu), which has a very good user interface as well as efficient methods for computing and analyzing the strategies. Another one is Elvira (http://leo.ugr.es/elvira), which is mostly focused on research purposes although it offers a clear user interface and a complete set of algorithms for inference on IDs and Bayesian networks.

Unfortunately, most of the models considered here are so recent that they are not yet incorporated in these tools. As far as we know, there are no commercial tools for evaluating or modeling with sequential decision diagrams, unconstrained influence diagrams, sequential valuation networks and sequential influence diagrams. HUGIN has incorporated LIMIDs and continuous variables, and Elvira offers algorithms for using MTEs for Bayesian networks inference. Therefore, this is an urgent need if we want these models to be widely used.

In summary, it seems that IDs have been accepted as a model for dealing with decision problems in many different fields such as business, chemistry, environmental science, and medicine. There is a general agreement on their capability for offering a clear and powerful representation for reasoning and decision making under uncertainty. 


\section{Summary and Discussion}

IDs have proven to be a powerful tool for communicating ideas in decision-making problems. IDs represent a new dimension for decision models [29]. As an extension of Bayesian networks, IDs have benefitted from research in artificial intelligence, decision analysis, and statistics. The intersection of these disciplines has produced a useful instrument for representing and solving decision-making problems. The analysis presented here reveals complementary cross-discipline efforts and interactions.

We have studied the qualitative level of representation of an ID. Several new capacities have been identified. Unlike traditional symmetric IDs, intelligent modifications of IDs allow one to directly model different kind of asymmetries - chance, decision and information asymmetries - in different ways. In Section 2, we have elaborated on conditional distribution trees, sequential decision diagrams, and sequential valuation networks, as the most outstanding approaches and mentioned a number of other graphical representations proposed in the literature. However, challenges to be able to model, e.g., constraints on the sequence of decisions remain.

Directly related to this, and more generally having different information constraints in all scenarios, led us to study, in Section 3, the contributions made to the so-called sequencing constraint. Examples include diagnosis/troubleshooting problems. The main models here are the unconstrained IDs, the sequential valuation network and the sequential IDs, where the requirement of a directed path including all decision variables is dropped. Sequential influence diagram representation is very efficient. However, when the number of decisions without constraints is large, solving these models may be intractable. The limited memory influence diagrams drop the no-forgetting condition, which may make problems that are intractable to solve exactly tractable for an approximate solution. The challenge here is, perhaps, to design "anytime" algorithms to find an approximate solution when having time constraints that prevent completion of an exact algorithm.

We have discussed some literature and challenges associated with the use of continuous chance and decision variables in IDs. This is an area that is ripe for further research on the use of mixture of truncated exponentials (MTE) functions and mixture of polynomials (MOP) functions to approximate PDFs and utility functions. Using the extended Shenoy-Shafer architecture [50], the MTE and MOP functions can be propagated in a binary join tree [79] to compute an optimal strategy.

We have not gone into other issues that are also important and are listed as follows:

- Decisions may involve groups of people rather than single decision makers [66, 87, 44, 21, 72].

- Beyond the typical what-if questions on the quantitative values of the ID, perturbations of the ID may also include its structure, i.e., critical evaluation of functional relations on variables is also necessary. This also involves the complicated and burdensome process of generating an ID. Ideas 
here range from suggesting a case-based reasoning approach where candidate IDs are retrieved from a set of similar IDs (a case base) [48], to using a neural network for yielding an initial ID that is later refined to generate a well-formed ID [43].

- Explanation capabilities of IDs are a key element that can improve the effectiveness and justification of decisions. Explanations enhance the decision maker's ability to understand and have confidence in the ID model, thereby accepting ID advices. Generating explanations is related to the search of the most relevant information and how to present it to the decision maker in a structured form. Although in Bayesian networks there has been much research in the topic, see a review in [45], we expect to see more work in decision-making [41, 5, 28].

- Modeling temporal processes is one of the greatest challenges of IDs. Dynamic IDs [86], and temporal IDs [67], are possibilities that have in (partially observable) Markov decision processes a strong competitor.

- The need to deal with time- or modeling-pressured situations in developing time-critical dynamic decision support systems is a practically useful but difficult problem. Xiang and Poh [88, 89] consider time-critical dynamic IDs to choose, from a meta-reasoning approach, the best model with optimal trade-off between model quality and computational tractability. Other authors construct anytime algorithms providing approximate strategies incrementally refined as computation progresses [30, 31, 32, 63, 70, 33].

- A daunting challenge would be to face the continuous change in the environment. IDs are difficult to scale up and adapt to changing and evolving knowledge, policies, standards, regulations, operating procedures, etc. Perhaps IDs were not conceived to reach so far. However, combining the inclusion of time together with a mechanism that detects when the ID fails and suggests an alternative model is a possibility. These adaptive models have been the topic of many recent world conferences, like that organized by AAAI in 2005.

- Moreover, models need to effectively operate in the rapidly developing distributed infrastructures, gathering knowledge from different sources [73], like from different computers in a network.

Acknowledgments. We are very grateful for discussions with T.D. Nielsen and J.Q. Smith during the workshop on "Graphical Modelling of Dependent Uncertainties for Decision Support in Public Policy" in August 2004. Thanks also to T. Bedford who convinced C. Bielza to prepare a talk in that workshop about challenges in large decision-making problems that served as the seed of this paper.

Research partially supported by the Spanish Ministry of Education and Science, projects TIN200762626 and TIN2007-67418-C03-03. 


\section{References}

[1] C. Bielza, J. Fernández del Pozo, and P. Lucas. Explaining clinical decisions by extracting regularity patterns. Decision Support Systems, 44:397-408, 2008.

[2] C. Bielza, M. Gómez, S. Ríos-Insua, and J. Fernández del Pozo. Structural, elicitation and computational issues faced when solving complex decision problems with influence diagrams. Computers and Operations Research, 27(7-8):725-740, 2000.

[3] C. Bielza, P. Müller, and D. Ríos. Decision analysis by augmented probability simulation. Management Science, 45(7):995-1007, 1999.

[4] C. Bielza and P. P. Shenoy. A comparison of graphical techniques for asymmetric decision problems. Management Science, 45(11):1552-1569, 1999.

[5] M. Bohanec and V. Rajkovic. Knowledge-based explanation in multiattribute decision making. In Computer aided decision analysis: Theory and applications, pages 189-204. Westport: Quorum Books, 1993.

[6] C. Boutilier. The influence of influence diagrams on artificial intelligence. Decision Analysis, 2(4):229-231, 2005.

[7] D. Buede. Influence diagrams: A practitioner's perspective. Decision Analysis, 2(4):235-237, 2005.

[8] H. Call and W. Miller. A comparison of approaches and implementations for automating decision analysis. Reliability Engineering and System Safety, 30:115-162, 1990.

[9] Y. B. Canbolat, K. Chelst, and N. Garg. Combining decision tree and MAUT for selecting a country for a global manufacturing facility. Omega, 35(3):312-325, 2007.

[10] J. M. Charnes and P. P. Shenoy. Multi-stage Monte Carlo method for solving influence diagrams using local computation. Management Science, 50(3):405-418, 2004.

[11] E. N. Cinicioglu and P. P. Shenoy. Arc reversals in hybrid Bayesian networks with deterministic variables. International Journal of Approximate Reasoning, 50(5):763-777, 2009.

[12] R. Clemen. Making Hard Decisions: An Introduction to Decision Analysis. South-Western College Pub., 1997.

[13] B. R. Cobb. Influence diagrams with continuous decision variables and non-gaussian uncertainties. Decision Analysis, 4(3):136-155, 2007. 
[14] B. R. Cobb and P. P. Shenoy. Hybrid Bayesian networks with linear deterministic variables. In F. Bacchus and T. Jaakkola, editors, Uncertainty in Artificial Intelligence: Proceedings of the 21st Conference, pages 136-144, Corvallis, OR, 2005. AUAI Press.

[15] B. R. Cobb and P. P. Shenoy. Nonlinear deterministic relationships in Bayesian networks. In L. Godo, editor, Symbolic and Quantitative Approaches to Reasoning with Uncertainty: 8th European Conference, ECSQARU 2005, Lecture Notes in Artificial Intelligence 3571, pages 27-38, Berlin, 2005. Springer.

[16] B. R. Cobb and P. P. Shenoy. Decision making with hybrid influence diagrams using mixtures of truncated exponentials. European Journal of Operational Research, 186(1):261-275, 2008.

[17] B. R. Cobb, P. P. Shenoy, and R. Rumi. Approximating probability density functions in hybrid Bayesian networks with mixtures of truncated exponentials. Statistics $\&$ Computing, 16(3):293$308,2006$.

[18] G. Cooper. A method for using belief networks as influence diagrams. In Proceedings of the 4th Conference on Uncertainty in Artificial Intelligence, pages 55-63, Minneapolis, 1988. University of Minnesota.

[19] Z. Covaliu and R. Oliver. Representation and solution of decision problems using sequential decision diagrams. Management Science, 41(12):1860-1881, 1995.

[20] R. Demirer and P. P. Shenoy. Sequential valuation networks for asymmetric decision problems. European Journal of Operational Research, 169(1):286-309, 2006.

[21] A. Detwarasiti and R. D. Shachter. Influence diagrams for team decision analysis. Decision Analysis, 2(4):207-228, 2005.

[22] P. A. M. Dirac. The physical interpretation of the quantum dynamics. Proceedings of the Royal Society of London, Series A, 113(765):621-641, 1927.

[23] J. Fernández del Pozo and C. Bielza. An interactive framework for open queries in decision support systems. In F. Garijo, J. Riquelme, and M. Toro, editors, Advances in Artificial IntelligenceIBERAMIA 2002, Lecture Notes in Artificial Intelligence 2527, pages 254-264, Berlin, 2002. Springer.

[24] J. Fernández del Pozo, C. Bielza, and M. Gómez. A list-based compact representation for large decision tables management. European Journal of Operational Research, 160(3):638-662, 2005. 
[25] R. M. Fung and R. D. Shachter. Contingent influence diagrams. Technical report, Department of Engineering-Economic Systems, Stanford Univerity, Stanford, Calif, 1990.

[26] M. Gómez. Real-world applications of influence diagrams. In J. Gámez, S. Moral, and A. Salmeron, editors, Advances in Bayesian Networks, Studies in Fuzziness and Soft Computing 146, pages 161180. Springer-Verlag, Berlin, 2004.

[27] M. Gómez, C. Bielza, J. Fernández del Pozo, and S. Ríos-Insua. A graphical decision-theoretic model for neonatal jaundice. Medical Decision Making, 27:250-265, 2007.

[28] M. Gönül, D. Önkal, and M. Lawrence. The effects of structural characteristics of explanations on use of a DSS. Decision Support Systems, 42:1481-1493, 2006.

[29] M. Helfand and S. Pauker. Influence diagrams: A new dimension for decision models. Medical Decision Making, 17(3):351-352, 1997.

[30] M. Horsch and D. Poole. Flexible policy construction by information refinement. In E. Horvitz and F. V. Jensen, editors, Uncertainty in Artificial Intelligence: Proceedings of the 12th Conference, pages 315-324, San Francisco, CA, 1996. Morgan Kaufmann.

[31] M. Horsch and D. Poole. An anytime algorithm for decision making under uncertainty. In G. Cooper and S. Moral, editors, Uncertainty in Artificial Intelligence: Proceedings of the 14th Conference, pages 246-255, San Francisco, 1998. Morgan Kaufmann.

[32] M. Horsch and D. Poole. Estimating the value of computation in flexible information refinement. In K. Laskey and H. Prade, editors, Uncertainty in Artificial Intelligence: Proceedings of the 15th Conference, pages 297-304, San Francisco, CA, 1999. Morgan Kaufmann.

[33] E. Horvitz and A. Seiver. Time-critical action: Representations and application. In D. Geiger and P. P. Shenoy, editors, Uncertainty in Artificial Intelligence: Proceedings of the 13th Conference, pages 250-257, San Francisco, CA, 1997. Morgan Kaufmann.

[34] R. Howard and J. Matheson. Influence diagrams. In R. Howard and J. Matheson, editors, Readings on the Principles and Applications of Decision Analysis, vol II, pages 719-762. Strategic Decisions Group, 1984.

[35] R. Howard and J. Matheson. Influence diagram retrospective. Decision Analysis, 2:144-147, 2005.

[36] R. Howard and J. Matheson. Influence diagrams. Decision Analysis, 2:127-143, 2005.

[37] R. Howard, J. Matheson, M. Merkhofer, A. Miller, and D. North. Comment on influence diagram retrospective. Decision Analysis, 3:117-119, 2006. 
[38] F. Jensen, F. V. Jensen, and D. Dittmer. From influence diagrams to junction trees. In R. de Mantaras and D. Poole, editors, Uncertainty in Artificial Intelligence: Proceedings of the 10th Conference, pages 367-373, San Francisco, CA, 1994. Morgan Kaufmann.

[39] F. V. Jensen, T. D. Nielsen, and P. P. Shenoy. Sequential influence diagrams: A unified asymmetry framework. International Journal of Approximate Reasoning, 42(1-2):101-118, 2006.

[40] F. V. Jensen and M. Vomlelova. Unconstrained influence diagrams. In A. Darwiche and N. Friedman, editors, Uncertainty in Artificial Intelligence: Proceedings of the 18th Conference, pages 234-241, San Francisco, CA, 2002. Morgan Kaufmann.

[41] H. Jimison, L. Fagan, R. Shachter, and E. Shortliffe. Patient-specific explanation in models of chronic disease. Artificial Intelligence in Medicine, 4:191-205, 1992.

[42] C. R. Kenley. Influence diagram models with continuous variables. PhD thesis, Stanford University, Stanford, CA., 1986.

[43] J. Kim, K. Lee, and J. Lee. Hybrid of neural network and decision knowledge approach to generating influence diagrams. Expert Systems with Applications, 23(3):237-244, 2002.

[44] D. Koller and B. Milch. Multi-agent influence diagrams for representing and solving games. Games and Economic Behavior, 45(1):181-221, 2003.

[45] C. Lacave and F. Díez. A review of explanation methods for heuristic expert systems. Knowledge Engineering Review, 19:133-146, 2004.

[46] S. L. Lauritzen and F. V. Jensen. Stable local computation with conditional Gaussian distributions. Statistics and Computing, 11:191-203, 2001.

[47] S. L. Lauritzen and D. Nilsson. Representing and solving decision problems with limited information. Management Science, 47(9):1235-1251, 2001.

[48] J. Lee, J. Kim, and S. Kim. A methodology for modeling influence diagrams: A case-based reasoning approach. International Journal of Intelligent Systems in Accounting, Finance 6 Management, $9(1): 55-63,2000$.

[49] U. Lerner, E. Segal, and D. Koller. Exact inference in networks with discrete children of continuous parents. In J. Breese and D. Koller, editors, Uncertainty in Artificial Intelligence: Proceedings of the 17th Conference, pages 319-328, San Francisco, CA, 2001. Morgan Kaufmann. 
[50] Y. Li and P. P. Shenoy. Solving hybrid influence diagrams with deterministic variables. In P. Grünwald and P. Spirtes, editors, Uncertainty in Artificial Intelligence: Proceedings of the 26th Conference (UAI-2010), pages 322-331, Corvallis, OR, 2010. AUAI Press.

[51] L. Liu and P. P. Shenoy. Representing asymmetric decision problems using coarse valuations. Decision Support Systems, 37(1):119-135, 2004.

[52] A. L. Madsen and F. V. Jensen. Lazy evaluation of symmetric Bayesian decision problems. In K. Laskey and H. Prade, editors, Uncertainty in Artificial Intelligence: Proceedings of the 15th Conference, pages 382-390, San Francisco, CA, 1999. Morgan Kaufmann.

[53] S. Moral, R. Rumí, and A. Salmerón. Mixtures of truncated exponentials in hybrid Bayesian networks. In S. Benferhat and P. Besnard, editors, Symbolic and Quantitative Approaches to Reasoning with Uncertainty: 6th European Conference, ECSQARU-2001, Lecture Notes in Artificial Intelligence 2143, pages 156-167, Berlin, 2001. Springer.

[54] K. Murphy. A variational approximation for Bayesian networks with discrete and continuous latent variables. In K. Laskey and H. Prade, editors, Uncertainty in Artificial Intelligence: Proceedings of the 15th Conference, pages 457-466, San Francisco, CA, 1999. Morgan Kaufmann.

[55] R. Nease and D. Owens. Use of influence diagrams to structure medical decisions. Medical Decision Making, 17:263-275, 1997.

[56] T. D. Nielsen and F. V. Jensen. Welldefined decision scenarios. In K. B. Laskey and H. Prade, editors, Uncertainty in Artificial Intelligence: Proceedings of the 15th Conference, pages 502-511, San Francisco, CA, 1999. Morgan Kaufmann.

[57] T. D. Nielsen and F. V. Jensen. Representing and solving asymmetric decision problems. International Journal of Information Technology and Decision Making, 2(2):217-263, 2003.

[58] D. North, A. Miller, and T. Braunstein. Decision analysis of intelligence resource allocation. Technical Report DAH C15-73-C-0430, Stanford Research Institute, Menlo Park, CA, 1974.

[59] S. Olmsted. On representing and solving decision problems. PhD thesis, Department of Engineering-Economic Systems, Stanford, CA., 1983.

[60] D. Owens, R. Shachter, and R. Nease. Representation and analysis of medical decision problems with influence diagrams. Medical Decision Making, 17:241-262, 1997.

[61] S. Pauker and J. Wong. The influence of influence diagrams in medicine. Decision Analysis, $2(4): 238-244,2005$. 
[62] J. Pearl. Influence diagrams-Historical and personal perspectives. Decision Analysis, 2:232-234, 2005.

[63] K. Poh and E. Horvitz. Reasoning about the value of decision-model refinement: Methods and application. In D. Heckerman and A. Mamdani, editors, Uncertainty in Artificial Intelligence: Proceedings of the 11th Conference, pages 174-182, San Francisco, CA, 1993. Morgan Kaufmann.

[64] W. B. Poland III. Decision analysis with continuous and discrete variables: A mixture distribution approach. PhD thesis, Stanford University, Stanford, CA., 1994.

[65] W. B. Poland III and R. D. Shachter. Mixtures of Gaussians and minimum relative entropy techniques for modeling continuous uncertainties. In D. Heckerman and A. Mamdani, editors, Uncertainty in Artificial Intelligence: Proceedings of the 9th Conference, pages 183-190, San Francisco, CA, 1993. Morgan Kaufmann.

[66] D. Poole. The independent choice logic for modelling multiple agents under uncertainty. Artificial Intelligence, 94:7-56, 1997.

[67] G. Provan and J. Clarke. Dynamic network construction and updating techniques for the diagnosis of acute abdominal pain. IEEE Transactions on Pattern Analysis and Machine Intelligence, 15(3):299-307, 1993.

[68] R. Qi, N. Zhang, and D. Poole. Solving asymmetric decision problems with influence diagrams. In R. de Mantaras and D. Poole, editors, Uncertainty in Artificial Intelligence: Proceedings of the 10th Conference, pages 491-497, San Francisco, CA, 1994. Morgan Kaufmann.

[69] H. Raiffa. Decision Analysis. Addison-Wesley, Reading, MA, 1968.

[70] M. Ramoni. Anytime influence diagrams. In Proceedings of the IJCAI Workshop on Anytime Algorithms and Deliberation Scheduling, pages 55-62. American Association for Artificial Intelligence, 1995.

[71] C. R. Rao. Linear Statistical Inference and Its Applications. Wiley Series in Probability and Mathematical Statistics. Wiley, 2nd edition edition, 1973.

[72] J. Ríos and D. Ríos-Insua. Negotiation over influence diagrams. Technical report, Rey Juan Carlos University, 2006.

[73] C. Schneeweiss. Distributed Decision Making. Springer, New York, 2nd edition edition, 2003.

[74] R. D. Shachter. Evaluating influence diagrams. Operations Research, 34:871-882, 1986. 
[75] R. D. Shachter. Efficient value of information computation. In K. B. Laskey and H. Prade, editors, Uncertainty in Artificial Intelligence: Proceedings of the 15th Conference, pages 594-602, San Francisco, CA, 1999. Morgan Kaufmann.

[76] R. D. Shachter and C. R. Kenley. Gaussian influence diagrams. Management Science, 35(5):527$550,1989$.

[77] R. D. Shachter and P. P. Ndilikilikesha. Using potential influence diagrams for probabilistic inference and decision making. In D. Heckerman and A. Mamdani, editors, Uncertainty in Artificial Intelligence: Proceedings of the 15th Conference, pages 383-390, San Francisco, CA, 1993. Morgan Kaufmann.

[78] P. P. Shenoy. Valuation-based systems for Bayesian decision analysis. Operations Research, 40(3):463-484, 1992.

[79] P. P. Shenoy. Binary join trees for computing marginals in the Shenoy-Shafer architecture. International Journal of Approximate Reasoning, 17(2-3):239-263, 1997.

[80] P. P. Shenoy. Valuation network representation and solution of asymmetric decision problems. European Journal of Operational Research, 121(3):579-608, 2000.

[81] P. P. Shenoy. Inference in hybrid Bayesian networks using mixtures of Gaussians. In R. Dechter and T. Richardson, editors, Uncertainty in Artificial Intelligence: Proceedings of the 22nd Conference, pages 428-436, Corvallis, OR, 2006. AUAI Press.

[82] P. P. Shenoy and J. C. West. Inference in hybrid Bayesian networks using mixtures of polynomials. Working Paper 321, University of Kansas School of Business, Lawrence, KS, May 2009.

[83] P. P. Shenoy and J. C. West. Inference in hybrid Bayesian networks with deterministic variables. In C. Sossai and G. Chemello, editors, Symbolic and Quantitative Approaches to Reasoning with Uncertainty-10th ECSQARU, Lecture Notes in Artificial Intelligence 5590, pages 46-58, Berlin, 2009. Springer-Verlag.

[84] P. P. Shenoy and J. C. West. Mixtures of polynomials in hybrid Bayesian networks with deterministic variables. In T. Kroupa and J. Vejnarova, editors, Proceedings of the 8th Workshop on Uncertainty Processing, pages 202-212, Prague, Czech Republic, 2009. University of Economics.

[85] J. Smith, S. Holtzman, and J. Matheson. Structuring conditional relationships in influence diagrams. Operations Research, 41(2):280-297, 1993. 
[86] J. A. Tatman and R. D. Shachter. Dynamic programming and influence diagrams. IEEE Transactions on Systems, Man and Cybernetics, 20(2):365-379, 1990.

[87] Y. Xiang. Probabilistic Reasoning in Multiagent Systems: A Graphical Models Approach. Cambridge University Press, 2002.

[88] Y. Xiang and K. Poh. Time-critical dynamic decision making. In K. Laskey and H. Prade, editors, Uncertainty in Artificial Intelligence: Proceedings of the 15th Conference, pages 688-695, San Francisco, CA, 1999. Morgan Kaufmann.

[89] Y. Xiang and K. Poh. Time-critical dynamic decision modeling in medicine. Computers in Biology and Medicine, 32(2):85-97, March 2002.

[90] N. L. Zhang. Probabilistic inference in influence diagrams. In G. Cooper and S. Moral, editors, Uncertainty in Artificial Intelligence: Proceedings of the 14th Conference, pages 514-522, San Francisco, CA, 1998. Morgan Kaufmann. 